\title{
Catheter ablation for AF improves global thrombotic profile and enhances fibrinolysis
}

\author{
Maria Niespialowska-Steuden ${ }^{1,2} \cdot V^{1 a s}$ Markides $^{1,3,4} \cdot$ Mohamed Farag $^{2,3} \cdot$ \\ David Jones $^{1,4}$ - Wajid Hussain ${ }^{1,4}$ • Tom Wong ${ }^{1,4}$ - Diana A. Gorog ${ }^{1,2,3}$
}

Published online: 18 September 2017

(C) The Author(s) 2017. This article is an open access publication

\begin{abstract}
Patients with atrial fibrillation (AF) are at increased risk of thrombotic events despite oral anticoagulation (OAC). Radiofrequency catheter ablation (RFCA) can restore and maintain sinus rhythm (SR) in patients with AF. To assess whether RFCA improves thrombotic status. 80 patients ( $71 \%$ male, $64 \pm 12 \mathrm{y}$ ) with recently diagnosed AF, on OAC and scheduled to undergo RFCA or DC cardioversion (DCCV) were recruited. Thrombotic status was assessed using the point-of-care global thrombosis test (GTT), before, and 4-6 weeks after DCCV and 3 months after RFCA. The GTT first measures the time taken for occlusive thrombus formation (occlusion time, OT), while the second phase of the test measures the time taken to spontaneously dissolve this clot through endogenous thrombolysis (lysis time, LT). 3 months after RFCA, there was a significant reduction in LT $(1994$ s [1560; 2475] vs. 1477s [1015; 1878]) in those who maintained SR, but not in those who reverted to AF. At follow-up, LT was longer in those in AF compared to those in SR (AF 2966s [2038; 3879] vs. SR 1477s [1015; 1878]). RFCA resulted in no change in OT value, irrespective of
\end{abstract}

Electronic supplementary material The online version of this article (doi:10.1007/s11239-017-1548-3) contains supplementary material, which is available to authorized users.

Diana A. Gorog

d.gorog@imperial.ac.uk

1 National Heart \& Lung Institute, Imperial College, Dovehouse Street, London SW3 6LY, UK

2 Postgraduate Medical School, University of Hertfordshire, Hatfield, UK

3 Department of Cardiology, East and North Hertfordshire NHS Trust, Hertfordshire, UK

4 Royal Brompton \& Harefield NHS Foundation Trust, London, UK rhythm outcome. Similarly, there was no change in OT or $\mathrm{LT}$ in response to DCCV, irrespective of whether SR was restored. Successful restoration and maintenance of SR following RFCA of AF is associated with improved global thrombotic status with enhanced fibrinolysis. Larger studies are required to confirm these early results and investigate whether improved thrombotic status translates into fewer thromboembolic events.

Keywords Atrial fibrillation - Thrombosis · Fibrinolysis · Ablation · Cardioversion

\section{Introduction}

Patients with atrial fibrillation (AF) exhibit a prothrombotic state and are at increased risk of thromboembolic events, which is reduced but not abolished by oral anticoagulation (OAC) [1, 2]. Left atrial thrombus formation in patients with AF is thought to be determined by the components of Virchow's triad, namely blood stasis (due to loss of atrial contractility and dilatation of the left atrium), endothelial injury (through myocyte hypertrophy, sclerosis and fibroelastosis) and a prothrombotic/hypercoagulable state [2, 3]. Endogenous fibrinolysis is a natural defense mechanism against lasting thrombus formation, such that the occurrence of thrombus is determined by the balance between procoagulant factors on the one hand, and effectiveness of fibrinolysis on the other. Radiofrequency catheter ablation (RFCA) can restore and maintain sinus rhythm (SR) longterm in patients with AF, with far superior efficacy compared to DCCV. Given that blood stasis due to reduced atrial contractility is an important component of Virchow's triad, it could be postulated that restoration of atrial contraction in AF patients through restoration of SR with RFCA may 
favourably reduce the prothrombotic state. It is acknowledged that additional factors including systemic inflammation may also contribute to a procoagulant state, which is one of the reasons for the current recommendation for continued anticoagulant therapy after successful RFCA. Superiority of RFCA, compared to a rate-control strategy, in improving symptoms and quality of life as well as the haemodynamic benefit in patients with heart failure, is well documented [4, 5]. However, whether maintenance of SR in AF patients can reduce thrombotic risk, is incompletely understood. Large studies, most notably the AFFIRM (atrial fibrillation follow-up investigation of rhythm management) trial, have not shown a mortality or stroke reduction benefit with rhythm control (achieved with antiarrhythmic medications and cardioversion) compared to rate control [6]. However, many more patients in the rhythm control arm had discontinued anticoagulation and a post-hoc analysis indicated a trend for mortality benefit following restoration of SR [7]. Non-randomized data indicate normalization of stroke risk following successful AF ablation, suggesting that treating the substrate of AF may favourably improve thrombotic profile [8]. The prothrombotic/hypercoagulable state in AF comprises enhanced platelet activation, evidenced by raised levels of soluble P-selectin, $\beta$-thromboglobulin and platelet factor four released from platelet alpha granules; as well as raised levels of von Willebrand factor released by the endothelium, which mediate subendothelial platelet adhesion; enhanced coagulation, through increased levels of prothrombin fragments 1 and 2 and thrombin-antithrombin III complex; and impaired fibrinolysis [3, 9]. The fibrinolytic system plays an important role in regulating local thrombus formation. Increased level of tissue-type plasminogen activator antigen and tissue-type plasminogen activator inhibitor-1 has been documented in AF [9] which is reduced significantly with anticoagulation [9]. D-dimer levels, reflecting fibrin turnover, are elevated in patients with AF, especially those with left atrial appendage thrombi and are reduced by anticoagulation [10]. We aimed to assess whether restoration of SR in patients with AF may have favourable effects on thrombotic profile, and whether modification of the substrate of $\mathrm{AF}$, through ablation improves thrombotic status more than simple restoration of SR through cardioversion. We used a method to assess thrombotic status that would capture changes in platelet activation, coagulation, and fibrinolysis, which are felt to belie the prothrombotic/hypercoagulable state in AF. To further assess whether changes in thrombotic status were systemic or local to the left atrium, we compared blood from the right and left atria with peripheral blood, in patients with and without AF undergoing ablation.

\section{Methods}

\section{Patients, clinical procedures and follow-up}

The study was approved by the National Research Ethics Service. 80 patients with AF scheduled to undergo direct current cardioversion (DCCV) $(n=40)$ or RFCA $(n=40)$ were enrolled after obtaining written informed consent. The exclusion criteria in Table 1 were applied. Venous blood was taken at 2 time-points from each patient; at baseline and at post-procedural follow up. Patients acted as self-controls.

Baseline demographics and clinical characteristics were obtained from patients, review of case notes and electronic patient records, at baseline and at follow-up, which was approximately 4-6 weeks after DCCV and 3 months after RFCA. The end-study visit involved assessment of thrombotic status, review of the 24-h Holter monitor and ECG results, and verification of other clinical characteristics.

Patients had been therapeutically anticoagulated for at least 4 weeks pre-DCCV. Non-vitamin $\mathrm{K}$ oral anticoagulants (NOAC) were taken on the day of the procedure. Cardioversion was performed according to standard local protocol under general anesthesia, delivering up to three biphasic synchronized shocks. 29 patients were successfully cardioverted to SR. Digoxin was stopped if SR was restored, but

Table 1 Exclusion criteria

\section{Exclusion criteria}

Age $<18$ years

Impaired renal function with eGFR $<30 \mathrm{~mL} / \mathrm{min}$ (since renal failure is associated with platelet function defect that may confound results)

Persons who, in the opinion of the investigator, have significant neurological, hepatic, renal, endocrine, cardiovascular, gastrointestinal, pulmonary, haemorrhagic, metabolic or other disease likely to confound the study results

Persons with a history of substance abuse or who demonstrates signs or clinical features of active substance abuse or psychiatric disease

Alcohol consumption above recommended safe levels (i.e. above 21 units per week for males, or 14 units per week for females) due to the potential effects of high alcohol levels on platelet reactivity

Any illness deemed significant by the investigator during the 4 weeks preceding the screening period of the study

Blood dyscrasia (platelets $<70 \times 10^{9} / 1, \mathrm{Hb}<80 \mathrm{~g} / \mathrm{dl}$, leucocyte count $<3.5 \times 10^{9} / 1$, neutrophil count $<1 \times 10^{9} / \mathrm{l}$ ), or known bleeding disorder

Subject currently enrolled in an investigational device or drug trial

$e G F R$ estimated glomerular filtration rate, $H b$ haemoglobin, $U N L$ upper normal limit 
other medications were continued. At 6 weeks' follow-up, 9 patients had AF recurrence, as evidenced by 24-h Holter monitoring and ECG. In patients taking warfarin, INR levels were similar before DCCV and at follow-up ( $2.5 \pm 0.33$ vs. $2.4 \pm 0.40 ; \mathrm{p}=0.24)$.

Patients undergoing RFCA had been therapeutically anticoagulated for at least 4 weeks. The morning dose of dabigatran was taken the morning of the day before the procedure. RFCA was performed according to standard local protocol under general anesthesia. All procedures were performed with a 3D mapping system, either CARTO3 (Biosense, Diamond Bar, CA, USA) or Velocity (St Jude Medical), alongside conventional mapping systems and irrigated ablation catheters. Wide area circumferential ablation with pulmonary vein isolation was performed in all patients, accompanied by linear ablation in the left and/or right atria with validation of line integrity, and limited electrogrambased ablation as clinically indicated. There were no major peri-procedural complications. No changes to medications were made post-procedure. At 3 months, AF recurred in 10 out of 40 RFCA patients. INR levels at baseline and followup were similar $(2.37 \pm 0.44$ vs. $2.33 \pm 0.40 ; p=0.67)$.

\section{Blood sampling}

Peripheral blood samples were taken on 2 occasions: (1) just before DCCV or RFCA, and (2) at follow-up, approximately 4-6 weeks after DCCV and 3 months after RFCA. Blood was obtained from an ante-cubital vein using an 18-G butterfly cannula with a two-syringe technique, taking care to avoid prolonged tourniquet time. The two-syringe technique involved discarding the first $5 \mathrm{~mL}$ of blood and using the second $5 \mathrm{~mL}$ for assessment of thrombotic status.

\section{Assessment of thrombotic status}

Assessment of thrombotic status was performed using the global thrombosis test (GTT) (Thromboquest Ltd., London, UK). This automated point-of-care test sequentially assesses both platelet reactivity and endogenous thrombolysis from a native blood sample. The patient was positioned in close proximity to the GTT such that the blood sample was injected into the instrument within $15 \mathrm{~s}$ of withdrawal and the automated measurement begun. The GTT assesses the time taken to form an occlusive platelet thrombus (occlusion time, OT), and in the second phase of the test, measures the time required to dissolve the thrombus formed in the first phase, through endogenous thrombolysis, which restores flow (lysis time, LT). The principle of the GTT has previously been described [11]. In brief, blood flows under physiological conditions through narrow gaps where it is subjected to high shear, and the instrument measures the time (d) between two consecutive blood drops downstream of this. As shear-induced platelet aggregation causes thrombus to form near the gaps, the time interval gradually increases as flow slows down and at an arbitrary point $(\mathrm{d} \geq 15 \mathrm{~s}$, before reaching complete occlusion), the end point of the measurement is displayed. The maximum time allowed for occlusive thrombus formation was pre-set to $800 \mathrm{~s}$. Following this, there is a preset "thrombus stabilization time" of $200 \mathrm{~s}$, during which the sensors are inactive. This time allows stabilization of the formed thrombi to allow lasting occlusion and ignores small re-bleeds. Restart of blood flow after occlusion is due to spontaneous thrombolysis. If lysis does not occur until >6000 s (LT cut-off time), "no lysis" is recorded. The coefficient of variation (cv) was assessed by testing a cohort of 10 stable patients on two occasions, $48 \mathrm{~h}$ apart.

\section{Chamber-specific assessment of thrombotic status}

In order to assess whether the prothrombotic state that exists in the left atrium of patients with AF is a local or systemic phenomenon, and to assess the chamber-specific differences in thrombotic status, in a separate group of 20 patients undergoing RFCA of AF or left atrial tachycardia for conventional clinical indications, thrombotic status was assessed in blood taken peripherally and from intra-cardiac chambers (Supplementary Table 1) at baseline before ablation. Blood samples were obtained from the femoral vein, right atrium and left atrium (prior to heparin administration) at the beginning of the procedure. Ablation was performed as above, according to standard protocols. Additional sampling was performed after heparin administration and at the end of the procedure (after administration of protamine to reverse heparin). Further samples were taken $4 \mathrm{~h}$ after ablation and at 3 months' follow-up from a peripheral vein.

\section{Statistical analysis}

Since this was a pilot study and patients acted as self-controls with pre- and post- intervention sampling to minimise any bias or confounders, samples of 40 patients per group were felt to be sufficient. Data are presented as mean and standard deviation for normally distributed or median [interquartile range] for skewed continuous variables and as proportions for categorical variables. Differences in OT/LT before and after DCCV/RFCA within one group were analyzed by paired t-test or Wilcoxon's method. Between group comparisons for continuous variables were assessed using a t-test or Mann-Whitney U test. The Kruskal-Wallis rank test was used to compare differences in baseline thrombotic status between patients with different types of AF. Dichotomous variables were compared using Chi square test with continuity correction or Fisher's exact test, as appropriate. Correlations were performed using Spearman's rank correlation. Univariate and multivariate linear regression models 
Table 2 Baseline characteristics of patients undergoing direct current cardioversion (DCCV) and radiofrequency ablation (RFCA), n (\%)

\begin{tabular}{|c|c|c|c|}
\hline Patient characteristic & DCCV cohort $(n=40)$ & RFCA cohort $(n=40)$ & $\mathrm{p}$ value \\
\hline Age, years & $63.9 \pm 11.6$ & $64 \pm 13$ & 0.534 \\
\hline Male & $29(72)$ & $28(70)$ & 0.851 \\
\hline BMI & $28.7 \pm 6$ & $28.5 \pm 5$ & 0.793 \\
\hline Paroxysmal AF & N/A & $10(25)$ & N/A \\
\hline Persistent AF & $19(48)$ & $9(23)$ & 0.034 \\
\hline Long-standing persistent AF & $21(53)$ & $21(53)$ & 1 \\
\hline \multicolumn{4}{|l|}{ Medical history } \\
\hline Hypertension & $22(55)$ & $11(27.5)$ & 0.023 \\
\hline Diabetes mellitus & $5(13)$ & $5(13)$ & 0.996 \\
\hline Hyperlipidemia & $18(45)$ & $17(42)$ & 0.851 \\
\hline Metabolic syndrome & $16(40)$ & $10(25)$ & 0.188 \\
\hline Coronary artery disease & $4(10)$ & $8(20)$ & 0.444 \\
\hline Prior stroke/TIA & $2(5)$ & $4(10)$ & 0.703 \\
\hline Chronic kidney disease $\left(\mathrm{eGFR}<60 \mathrm{~mL} / \mathrm{min} / 1.73 \mathrm{~m}^{2}\right)$ & $5(13)$ & $5(13)$ & 0.996 \\
\hline \multicolumn{4}{|l|}{ Echocardiographic characteristics } \\
\hline Ejection fraction $>55 \%$ & $20(50)$ & $30(75)$ & 0.284 \\
\hline Ejection fraction $45-54 \%$ & $18(45)$ & $7(18)$ & 0.067 \\
\hline Ejection fraction $<44 \%$ & $2(5)$ & $3(8)$ & 0.703 \\
\hline No atrial dilatation & $11(27)$ & $6(15)$ & 0.703 \\
\hline Mild atrial dilatation (LA diameter $4.1-4.6 \mathrm{~cm}$ ) & $18(45)$ & $25(63)$ & 0.179 \\
\hline Moderate atrial dilatation (LA diameter $4.7-5.2 \mathrm{~cm}$ ) & $9(23)$ & $5(13)$ & 0.179 \\
\hline Severe atrial dilatation (LA diameter $>5.3 \mathrm{~cm}$ ) & $2(5)$ & $4(10)$ & 0.703 \\
\hline \multicolumn{4}{|l|}{ Risk scores } \\
\hline $\mathrm{CHA}_{2} \mathrm{DS}_{2} \mathrm{VASc}$ & $1.9 \pm 1.39$ & $1.9 \pm 1.7$ & 0.798 \\
\hline HASBLED & $1.25 \pm 0.83$ & $1.2 \pm 0.7$ & 0.754 \\
\hline \multicolumn{4}{|l|}{ Medications } \\
\hline Warfarin & $20(50)$ & $36(90)$ & 0.116 \\
\hline Apixaban & $2(5)$ & $0(0)$ & N/A \\
\hline Rivaroxaban & $1(3)$ & $0(0)$ & N/A \\
\hline Dabigatran & $17(42)$ & $4(10)$ & 0.013 \\
\hline Beta-blocker & $31(77)$ & $21(53)$ & 0.179 \\
\hline Calcium channel blocker & $10(25)$ & $6(15)$ & 0.444 \\
\hline Digoxin & $6(15)$ & $1(2.5)$ & 0.338 \\
\hline Amiodarone & $1(3)$ & $6(15)$ & 0.338 \\
\hline Statin & $13(32)$ & $17(43)$ & 0.444 \\
\hline ACE inhibitor & $11(27)$ & $6(15)$ & 0.338 \\
\hline $\mathrm{ARB}$ & $8(20)$ & $6(15)$ & 0.703 \\
\hline \multicolumn{4}{|l|}{ Laboratory characteristics } \\
\hline Haemoglobin $(\mathrm{g} / \mathrm{L})$ & $144.6 \pm 15$ & $139 \pm 17$ & 0.186 \\
\hline Haematocrit (\%) & $0.43 \pm 0.04$ & $0.41 \pm 4$ & 0.159 \\
\hline Platelet count $\left(\times 10^{9} / \mathrm{L}\right)$ & $234 \pm 83$ & $212 \pm 58$ & 0.508 \\
\hline INR (warfarin patients) & $2.32 \pm 0.47$ & $2.3 \pm 0.4$ & 0.099 \\
\hline Creatinine $(\mu \mathrm{mol} / \mathrm{L})$ & $83.2 \pm 14.5$ & $95 \pm 84$ & 0.411 \\
\hline eGFR $\left(\mathrm{mL} / \mathrm{min} / 1.73 \mathrm{~m}^{2}\right)$ & $87.6 \pm 25$ & $96.6 \pm 28$ & 0.145 \\
\hline Total cholesterol (mmol/L) & $5.2 \pm 1.2$ & $5.0 \pm 1.7$ & 0.428 \\
\hline $\mathrm{CRP}(\mathrm{mg} / \mathrm{L})$ & $3.8 \pm 4.53$ & $3.52 \pm 4.18$ & 0.778 \\
\hline Fibrinogen $(\mathrm{g} / \mathrm{L})$ & $4.1 \pm 1.14$ & $4.06 \pm 1.64$ & 0.893 \\
\hline Baseline OT (s) & $488[396-573]$ & $524[445-641]$ & 0.123 \\
\hline Baseline LT (s) & 1819 [1453-2180] & 2015 [1507-2740] & 0.148 \\
\hline
\end{tabular}


Table 2 (continued)

Left ventricular ejection fraction was calculated by Simpson's method

$A C E$ angiotensin-converting enzyme, $A R B$ angiotensin receptor blocker, $B M I$ body mass index, $C R P$ C-reactive protein, $e G F R$ estimated glomerular rate, INR International Normalized Ratio, $L A$ left atrium, N/A not applicable, TIA transient ischaemic attack

Normal values: haemoglobin $130-180 \mathrm{~g} / \mathrm{L}$ in men and $115-165 \mathrm{~g} / \mathrm{L}$ in women; haematocrit $40-52 \%$ in men and $36-47 \%$ in women; platelet count $150-400 \times 10^{9} / \mathrm{L}$; INR range $2.0-3.0$; creatinine $60-110 \mu \mathrm{mol} / \mathrm{L}$ in men and $45-90 \mu \mathrm{mol} / \mathrm{L}$ in women; eGFR: above $90 \mathrm{~mL} / \mathrm{min} / 1.73 \mathrm{~m}{ }^{2}$; total cholesterol $<5.2 \mathrm{mmol} / \mathrm{L}$; CRP $0-10 \mathrm{mg} / \mathrm{L}$; fibrinogen $2-5 \mathrm{~g} / \mathrm{L}$

were used to identify the independent predictors of impaired fibrinolysis in the DCCV and ablation cohorts. All the study variables listed in Tables 2 and 3 were first analysed with univariate analysis and those that showed a significant interaction $(\mathrm{p}<0.05)$ in the univariate analysis were entered into the multivariate analysis. Statistical significance was fixed at 0.05. Analyses were performed with STATISTICA software (StatSoft, version 10).

\section{Results}

Clinical characteristics of the DCCV and RFCA cohorts are shown in Tables 2, 3 and 4. The cv for OT and LT was 8 and $10 \%$, respectively. The variables in Tables 2,3 and 4 were interrogated for relation to pre- and post-procedural OT and LT in each cohort. In the DCCV cohort, there was a trend for an inverse relationship between baseline OT and haemoglobin level $(r=-0.495 ; p=0.08)$ but not to other variables. In the RFCA cohort, there was a weak inverse correlation between baseline OT and BMI $(r=-0.358 ; p=0.025)$. Baseline LT correlated with creatinine $(r=0.331 ; p=0.044)$. LT after RFCA correlated with creatinine $(r=0.504 ; p=0.001)$ and weakly with haematocrit $(r=0.35 ; \mathrm{p}=0.049)$. Applying the Kruskal-Wallis rank test to the group as a whole (DCCV and RFA groups combined), we found that baseline OT was not different between patients with persistent AF, paroxysmal AF, and long-standing persistent AF (497 [402-571] vs. 640 [505-729] vs. 497 [420-603] respectively; $p=0.100$ ). Similarly, baseline LT did not differ between patients with different types of AF (1769 [1527-2217] vs. 2128 [1706-4537] vs. 1852 [1346-2228] respectively; $p=0.275)$.

\section{Effect of AF ablation on thrombotic status}

In the ablation cohort, 10 of the 40 patients had AF recurrence at follow-up, which was associated with longer ablation procedure duration $(\mathrm{p}=0.001)$, longer ablation time $(p=0.002)$, and higher energy application $(p=0.005)$ (Table 4).

In the group as a whole, there was no difference in OT before and after RFCA (523s [443; 640] vs. 571s [463; 682]; $\mathrm{p}=0.139$ ), regardless of whether patients were in AF at follow-up (493s [ $457 ; 691]$ vs. 552s [ $436 ; 685] ; p=0.514)$ or in SR (527s [440; 640] vs. 571s [476; 679]; $\mathrm{p}=0.135)$ (Figs. 1,
2). At follow-up, there was no difference in OT between patients in SR and those in AF (571 s [476; 679] vs. $552 \mathrm{~s}$ [436; 685]; $p=0.9)$.

In the RFCA cohort as a whole, LT was shorter after RFCA (2015s [1506; 2739] vs. 1645s [1037; 2285]; $\mathrm{p}=0.014$ ) (Figs. 1, 2). Among patients in SR at follow-up, there was a significant reduction in LT following RFCA (1994s [1560; 2475] vs. 1477s [1015; 1878]; $\mathrm{p}<0.001)$, whereas there was no change in LT in those in AF at followup $(2074$ s [1453; 3859] vs. 2966s [2038; 3879]; $p=0.500)$ (Figs. 2, 3). At follow-up, LT was significantly longer in those in AF compared to those in SR (2966s [2038; 3879] vs. $1477 \mathrm{~s}$ [1015; 1878]; $p=0.002$ ). Pre-ablation samples showed no baseline differences in LT between those who subsequently reverted to $\mathrm{AF}$ and those who maintained SR (2074s [1453; 3859] vs. 1994s [1560; 2475]; $p=0.606)$. Amongst patients undergoing RFCA, there was no difference between patients pre-and post-RFCA with respect to C-reactive protein (CRP, $3.52 \pm 4.18$ vs. $2.62 \pm 3.01, \mathrm{p}=0.279$ ) or fibrinogen $(4.06 \pm 1.64$ vs. $4.39 \pm 1.46, p=0.337)$. In the subgroup who reverted to $\mathrm{AF}$, there was no difference in CRP $(3.3 \pm 3.97$ vs. $3.2 \pm 3.15, \mathrm{p}=0.955)$ or fibrinogen $(4.35 \pm 1.32$ vs. $4.48 \pm 1.55, \mathrm{p}=0.847)$ before and after the procedure. In the subgroup who maintained SR, there was no difference in CRP $(3.6 \pm 4.31$ vs. $2.43 \pm 2.99 ; \mathrm{p}=0.223)$ or fibrinogen $(3.96 \pm 1.74$ vs. $4.36 \pm 1.46 ; \mathrm{p}=0.332)$ before and after the procedure.

In the ablation group, using univariate linear regression analysis, only the following variables were related to enhanced fibrinolysis (reduced delta LT): energy application $(p=0.039)$ and sinus rhythm at follow-up $(p=0.009)$. None of the other variables, including all clinical, procedural or haematological parameters listed in Tables 2 and 3, correlated with enhanced fibrinolysis. Multivariate analysis showed that sinus rhythm at follow-up remained a predictor of enhanced fibrinolysis after adjustment for other risk factors $(\mathrm{p}=0.028)$.

\section{Effect of cardioversion on thrombotic status}

In the cardioversion cohort, 20 of the 40 patients had reverted to $\mathrm{AF}$ at follow-up. In the group as a whole, there was no difference in OT before and after DCCV (median [interquartile range], 487s [395; 573] vs. 482s [398; 639]; $\mathrm{p}=0.247$ ), nor in the subgroup who were in AF at follow-up 
Table 3 Baseline clinical characteristics of direct current cardioversion (DCCV) cohort, with respect to rhythm outcome at follow-up

\begin{tabular}{|c|c|c|c|c|}
\hline Patient characteristic & DCCV cohort $(n=40)$ & AF at follow-up $(n=20)$ & SR at follow-up $(n=20)$ & $\overline{p \text { value }}$ \\
\hline Age, years & $63.9 \pm 11.6$ & $62.5 \pm 12$ & $65.2 \pm 11.5$ & 0.472 \\
\hline Male & $29(72 \%)$ & $15(75)$ & $14(70)$ & 0.731 \\
\hline BMI & $28.7 \pm 6$ & $29.6 \pm 7$ & $27.5 \pm 4.3$ & 0.358 \\
\hline \multicolumn{5}{|l|}{ Medical history } \\
\hline Hypertension & $22(55)$ & $12(60)$ & $10(50)$ & 0.537 \\
\hline Diabetes mellitus & $5(13)$ & $1(5)$ & $4(20)$ & 0.159 \\
\hline Hyperlipidemia & $18(45)$ & $7(35)$ & $11(55)$ & 0.213 \\
\hline Metabolic syndrome & $16(40)$ & $7(35)$ & $9(45)$ & 0.821 \\
\hline Coronary artery disease & $4(10)$ & $2(10)$ & $2(10)$ & 1 \\
\hline Prior stroke/TIA & $2(5)$ & $1(5)$ & $1(5)$ & 1 \\
\hline $\begin{array}{l}\text { Chronic kidney dis- } \\
\text { ease }(\mathrm{eGFR}<60 \mathrm{~mL} / \\
\left.\mathrm{min} / 1.73 \mathrm{~m}^{2}\right)\end{array}$ & $5(12.5)$ & $1(5)$ & $4(20)$ & 0.159 \\
\hline \multicolumn{5}{|l|}{ Echocardiographic characteristics } \\
\hline \multicolumn{5}{|l|}{ Left ventricular function } \\
\hline Ejection fraction $>55 \%$ & $20(50)$ & $9(45)$ & $11(55)$ & 0.539 \\
\hline Ejection fraction $45-54 \%$ & $18(45)$ & $9(45)$ & $9(45)$ & 1 \\
\hline Ejection fraction $<44 \%$ & $2(5)$ & $2(10)$ & $0(0)$ & 0.154 \\
\hline No atrial dilatation & $11(27)$ & $4(20)$ & $7(35)$ & 0.3 \\
\hline $\begin{array}{l}\text { Mild atrial dilatation (LA } \\
\text { diameter } 4.1-4.6 \mathrm{~cm} \text { ) }\end{array}$ & $18(45)$ & $9(45)$ & $9(45)$ & 1 \\
\hline $\begin{array}{l}\text { Moderate atrial dilatation (LA } \\
\text { diameter } 4.7-5.2 \mathrm{~cm} \text { ) }\end{array}$ & $9(22.5)$ & $6(30)$ & $3(15)$ & 0.267 \\
\hline $\begin{array}{l}\text { Severe atrial dilatation (LA } \\
\text { diameter }>5.3 \mathrm{~cm} \text { ) }\end{array}$ & $2(5)$ & $1(5)$ & $1(5)$ & 1 \\
\hline \multicolumn{5}{|l|}{ Risk scores } \\
\hline $\mathrm{CHA}_{2} \mathrm{DS}_{2} \mathrm{VASc}$ & $1.9 \pm 1.39$ & $1.9 \pm 1.2$ & $1.9 \pm 1.6$ & 1 \\
\hline HASBLED & $1.25 \pm 0.83$ & $1.3 \pm 0.8$ & $1.2 \pm 0.89$ & 0.711 \\
\hline \multicolumn{5}{|l|}{ Medications } \\
\hline Warfarin & $20(50 \%)$ & $9(45)$ & $11(55)$ & 0.539 \\
\hline Apixaban & $2(5)$ & $0(0)$ & $2(10)$ & N/A \\
\hline Rivaroxaban & $1(2.5)$ & $1(5)$ & 0 & N/A \\
\hline Dabigatran & $17(42)$ & $11(55)$ & $6(30)$ & 0.115 \\
\hline Beta-blocker & $31(77)$ & $15(75)$ & $16(80)$ & 0.713 \\
\hline Calcium channel blocker & $10(25)$ & $7(35)$ & $3(15)$ & 0.151 \\
\hline Digoxin & $6(15)$ & $4(20)$ & $2(10)$ & 0.388 \\
\hline Amiodarone & $1(2.5)$ & $0(0)$ & $1(5)$ & 0.323 \\
\hline Statin & $13(32)$ & $4(20)$ & $9(45)$ & 0.095 \\
\hline ACE inhibitor & $11(27)$ & $8(40)$ & $3(15)$ & 0.08 \\
\hline $\mathrm{ARB}$ & $8(20)$ & $5(25)$ & $3(15)$ & 0.692 \\
\hline \multicolumn{5}{|l|}{ Laboratory characteristics } \\
\hline Haemoglobin (g/L) & $144.6 \pm 15$ & $147 \pm 14$ & $141 \pm 16.7$ & 0.318 \\
\hline Haematocrit (\%) & $0.43 \pm 0.04$ & $0.43 \pm 0.04$ & $0.42 \pm 0.05$ & 0.692 \\
\hline Platelet count $\left(\times 10^{9} / \mathrm{L}\right)$ & $234 \pm 83$ & $251 \pm 101$ & $208 \pm 35.6$ & 0.192 \\
\hline INR (warfarin patients) & $2.54 \pm 0.33$ & $2.59 \pm 0.37$ & $2.47 \pm 0.28$ & 0.673 \\
\hline Creatinine $(\mu \mathrm{mol} / \mathrm{L})$ & $83.2 \pm 14.5$ & $85 \pm 9.5$ & $81.2 \pm 18$ & 0.382 \\
\hline eGFR (mL/min/1.73 m²) & $87.6 \pm 25$ & $85.8 \pm 21$ & $89 \pm 29$ & 0.661 \\
\hline Total cholesterol (mmol/L) & $5.2 \pm 1.2$ & $5.7 \pm 1.7$ & $4.8 \pm 0.5$ & 0.266 \\
\hline $\mathrm{CRP}(\mathrm{mg} / \mathrm{L})$ & $3.8 \pm 4.53$ & $4.25 \pm 4.71$ & $3.35 \pm 4.42$ & 0.537 \\
\hline Fibrinogen (g/L) & $4.1 \pm 1.14$ & $3.93 \pm 1.06$ & $4.28 \pm 1.21$ & 0.34 \\
\hline
\end{tabular}


Table 3 (continued)

Values are mean \pm standard deviation or $\mathrm{n}(\%)$. Left ventricular ejection fraction was calculated by Simpson's method

$A C E$ angiotensin-converting enzyme, $A R B$ angiotensin receptor blocker, $B M I$ body mass index, $C R P$ C-reactive protein, $e G F R$ estimated glomerular filtration rate, INR international normalized ratio, $L A$ left atrium, TIA transient ischaemic attack

Normal values: haemoglobin $130-180 \mathrm{~g} / \mathrm{L}$ in men and $115-165 \mathrm{~g} / \mathrm{L}$ in women; haematocrit $40-52 \%$ in men and $36-47 \%$ in women; platelet count $150-400 \times 10^{9} / \mathrm{L}$; INR range $2.0-3.0$; creatinine $60-110 \mu \mathrm{mol} / \mathrm{L}$ in men and $45-90 \mu \mathrm{mol} / \mathrm{L}$ in women; eGFR: above $90 \mathrm{~mL} / \mathrm{min}^{\prime} / 1.73 \mathrm{~m}{ }^{2}$; total cholesterol $<5.2 \mathrm{mmol} / \mathrm{L}$; CRP 0-10 $\mathrm{mg} / \mathrm{L}$; fibrinogen $2-5 \mathrm{~g} / \mathrm{L}$

(468s [392; 606] vs. 488s [414; 613]; $\mathrm{p}=0.601)$, nor in those in SR (496s [416; 573] vs. 447s [394; 652]; $\mathrm{p}=0.232)$ (Figs. 3, 4 and supplementary Fig. 5). At follow-up, there was no difference in OT between those patients who were in AF and those in SR (490s [394; 656] vs. 511s [398; 718]; $\mathrm{p}=0.786$ ).

There was no difference in LT before and after DCCV $(1819$ s [1453; 2180] vs. 2026s [1647; 2367]; $p=0.052)$. However, compared to baseline, those who were in AF at follow-up demonstrated longer LT after DCCV (1852s [1346; 2208] vs. 2156 s [1713; 2542]; $p=0.005)$, whereas there was no difference in LT before and after DCCV in those in SR at follow-up $(1711$ s [1579; 1951] vs. 1877s [1216; 2367]; $p=1.000$ ) (Fig. 4). At follow-up, there was no significant difference in LT between patients in AF and those in SR (2156s [1713; 2542] vs. 1877s [1216; 2367]; $p=0.14)$.

There was no difference between patients pre-and postDCCV with respect to CRP $(3.8 \pm 4.53$ vs. $4.46 \pm 6.19$; $\mathrm{p}=0.467)$ or fibrinogen $(4.1 \pm 1.14$ vs. $3.67 \pm 1.18$; $\mathrm{p}=0.115$ ). In the subgroup who reverted to AF, there was no difference in CRP $(4.25 \pm 4.71$ vs. $3.85 \pm 3.99 ; \mathrm{p}=0.776)$ or fibrinogen $(3.93 \pm 1.06$ vs. $3.77 \pm 1.38 ; \mathrm{p}=0.733)$ before and after the procedure; similarly, amongst those who maintained SR, there was no difference in either CRP $(3.35 \pm 4.42$ vs. $5.1 \pm 7.95 ; p=0.158)$ or fibrinogen $(4.28 \pm 1.21$ vs. $3.75 \pm 1.08 ; p=0.089$ ) before and after the procedure.

In the DCCV group, using univariate linear regression analysis, only the following variables were related to enhanced fibrinolysis (reduced delta LT): high platelet count $(p=0.035)$ and angiotensin-converting enzyme inhibitor treatment $(p=0.002)$. None of the other variables including all clinical, procedural or haematological parameters in Tables 2 and 3 correlated with enhanced fibrinolysis.

\section{Central and peripheral assessment of thrombotic status}

Patient and procedural characteristics are shown in Supplementary Table 1 and thrombotic status in Supplementary Table 2. Amongst patients with AF or left sided atrial tachycardia, there was no difference in OT or LT between blood samples from the femoral vein, right atrium or left atrium. Immediately after ablation, OT was significantly and profoundly prolonged, likely due to the effect of heparin, such that occlusive thrombus formation did not occur within the cut-off time of the test (800 s) and therefore LT also could not be measured (recorded as LT $>6000 \mathrm{~s}$ ). Ablation was not reflected in an acute change in OT or LT at $4 \mathrm{~h}$ after ablation compared to baseline, with the exception of AF patients, in whom heparin was administered after the baseline sample and where $4 \mathrm{~h}$ later, OT was prolonged, likely an effect of heparin, rather than the ablation.

\section{Discussion}

In this small exploratory study, successful treatment and restoration of SR in patients with AF using RFCA, is associated with improved global thrombotic status as evidenced by enhanced fibrinolysis. This improvement was not seen in patients who had restoration of SR using DCCV. Our study is hypothesis generating and suggests that altering the substrate of AF, through RFCA, not only improves the likelihood of maintaining SR, but may favourably alter thrombotic status.

A reduction of thrombotic risk would be expected with return of SR, in light of the restoration of effective mechanical atrial contraction and reduction in stasis. Prior studies have raised the possibility that AF ablation may reduce thrombotic risk [12, 13]. A registry comparing 4212 patients undergoing $\mathrm{AF}$ ablation to 16,848 matched controls with $\mathrm{AF}$ but without ablation, showed that AF patients treated with ablation had much lower risk of death, stroke, and dementia over 3-year follow-up, compared to AF patients without ablation [5, 8]. Furthermore, patients with AF ablation had similar long-term risk of stroke as patients without AF. This study however was not blinded or randomized, and the ablation arm had much more vigorous management than the control arm. A retrospective analysis of 24,244 patients with AF undergoing DCCV or RFA, showed that long term, ablation is associated with a lower rate of death, stroke or transient ischaemic attack than DCCV [12]. Two ongoing studies, the catheter ablation versus anti-arrhythmic drug therapy for atrial fibrillation trial (CABANA) trial (clinicaltrials.gov identifier NCT00911508), a prospective, randomized trial comparing RFCA with optimal medical therapy and EAST (early treatment of atrial fibrillation for stroke prevention trial, clinicaltrials.gov identifier NCT01288352) comparing "early" RFCA and antiarrhythmic medications vs. current 
Table 4 Baseline clinical characteristics of radiofrequency catheter ablation (RFCA) cohort with respect to rhythm at follow-up

\begin{tabular}{|c|c|c|c|c|}
\hline Patient characteristic & RFCA cohort $(n=40)$ & $\mathrm{AF}$ at follow-up $\mathrm{n}=10$ & SR at follow-up $n=30$ & $\mathrm{p}$ value \\
\hline Age, years & $64 \pm 13$ & $67 \pm 13$ & $63.8 \pm 14$ & 0.418 \\
\hline Male & $28(70)$ & $7(70)$ & $21(70)$ & 1.000 \\
\hline BMI & $28.5 \pm 5$ & $31.6 \pm 3.4$ & $27.6 \pm 5$ & 0.064 \\
\hline Paroxysmal AF & $10(25)$ & $3(30)$ & $7(23)$ & 0.766 \\
\hline Persistent AF & $9(23)$ & $3(30)$ & $6(20)$ & 0.416 \\
\hline Long-standing persistent AF & $21(53)$ & $4(40)$ & $17(57)$ & 0.444 \\
\hline \multicolumn{5}{|l|}{ Medical history } \\
\hline Duration of $\mathrm{AF}>1$ year & $29(72.5)$ & $7(70)$ & $22(73)$ & 1.000 \\
\hline Previous ablations & $13(32.5)$ & $5(50)$ & $8(26.7)$ & 0.479 \\
\hline Hypertension & $11(27.5)$ & $4(40)$ & $7(23)$ & 0.467 \\
\hline Diabetes mellitus & $5(12.5)$ & 0 & $5(16.7)$ & 0.571 \\
\hline Hyperlipidemia & $17(42)$ & $5(50)$ & $12(40)$ & 0.751 \\
\hline Metabolic syndrome & $10(25)$ & $4(40)$ & $6(18)$ & 0.014 \\
\hline Coronary artery disease & $8(20)$ & $2(20)$ & $6(20)$ & 1.000 \\
\hline Prior stroke/TIA & $4(10)$ & 0 & $4(13.3)$ & 0.559 \\
\hline Chronic kidney disease $\left(\mathrm{eGFR}<60 \mathrm{~mL} / \mathrm{min} / 1.73 \mathrm{~m}^{2}\right)$ & $5(12.5)$ & $2(20)$ & $3(10)$ & 0.598 \\
\hline \multicolumn{5}{|l|}{ Echocardiographic characteristics } \\
\hline Ejection fraction $>55 \%$ & $30(75)$ & $8(80)$ & $22(73.3)$ & 1.000 \\
\hline Ejection fraction $45-54 \%$ & $7(17.5)$ & $1(10)$ & $5(16.7)$ & 1.000 \\
\hline Ejection fraction $<44 \%$ & $3(7.5)$ & $1(10)$ & $3(10)$ & 1.000 \\
\hline No atrial dilatation & $6(15)$ & 0 & $9(30)$ & 0.173 \\
\hline Mild atrial dilatation (LA diameter $4.1-4.6 \mathrm{~cm}$ ) & $25(62.5)$ & $7(70)$ & $18(60)$ & 0.781 \\
\hline Moderate atrial dilatation (LA diameter $4.7-5.2 \mathrm{~cm}$ ) & $5(12.5)$ & $2(20)$ & 0 & 0.077 \\
\hline Severe atrial dilatation (LA diameter $>5.3 \mathrm{~cm}$ ) & $4(10)$ & $1(10)$ & $3(10)$ & 1.000 \\
\hline \multicolumn{5}{|l|}{ Risk scores } \\
\hline $\mathrm{CHA}_{2} \mathrm{DS}_{2}$-VASc & $1.9 \pm 1.7$ & $2.2 \pm 1.6$ & $1.83 \pm 1.8$ & 0.571 \\
\hline HASBLED & $1.2 \pm 0.7$ & $1.1 \pm 0.6$ & $1.2 \pm 0.7$ & 0.691 \\
\hline \multicolumn{5}{|l|}{ Medications } \\
\hline Warfarin & $36(90)$ & $9(90)$ & $27(90)$ & 1.000 \\
\hline Dabigatran & $4(10)$ & $1(10)$ & $3(10)$ & 1.000 \\
\hline Beta-blocker & $21(53)$ & $2(20)$ & $19(63)$ & 0.189 \\
\hline Calcium antagonist & $6(15)$ & $4(40)$ & $2(7)$ & 0.060 \\
\hline Digoxin & $1(3)$ & $1(10)$ & 0 & 0.268 \\
\hline Statin & $17(43)$ & $6(60)$ & $11(37)$ & 0.523 \\
\hline ACE inhibitor & $6(15)$ & $3(30)$ & $3(10)$ & 1.000 \\
\hline ARB & $6(15)$ & $1(10)$ & $5(17)$ & 1.000 \\
\hline \multicolumn{5}{|l|}{ Laboratory characteristics } \\
\hline Haemoglobin (g/L) & $139 \pm 17$ & $144 \pm 16$ & $137 \pm 15$ & 0.217 \\
\hline Haematocrit (\%) & $0.41 \pm 4$ & $0.42 \pm 6$ & $0.41 \pm 4$ & 0.326 \\
\hline Platelet count $\left(\times 10^{9} / \mathrm{L}\right)$ & $212 \pm 58$ & $227 \pm 52$ & $207 \pm 59$ & 0.244 \\
\hline INR (warfarin patients) & $2.3 \pm 0.4$ & $2.3 \pm 0.4$ & $2.3 \pm 0.4$ & 0.967 \\
\hline Creatinine $(\mu \mathrm{mol} / \mathrm{L})$ & $95 \pm 84$ & $93 \pm 28$ & $96 \pm 10$ & 0.137 \\
\hline $\mathrm{eGFR}\left(\mathrm{mL} / \mathrm{min} / 1.73 \mathrm{~m}^{2}\right)$ & $96.6 \pm 28$ & $85 \pm 21$ & $100.8 \pm 30$ & 0.35 \\
\hline Total cholesterol (mmol/L) & $5.0 \pm 1.7$ & $4.5 \pm 0.9$ & $5.2 \pm 1.9$ & 0.523 \\
\hline $\mathrm{CRP}(\mathrm{mg} / \mathrm{L})$ & $3.52 \pm 4.18$ & $3.3 \pm 3.97$ & $3.6 \pm 4.31$ & 0.847 \\
\hline Fibrinogen (g/L) & $4.06 \pm 1.64$ & $4.35 \pm 1.32$ & $3.96 \pm 1.74$ & 0.53 \\
\hline \multicolumn{5}{|l|}{ Procedure characteristics } \\
\hline Procedure duration (min) & $163 \pm 76$ & $247 \pm 64$ & $140 \pm 62$ & 0.001 \\
\hline Ablation time (ms) & $1856 \pm 1408$ & $3358 \pm 1451$ & $1446 \pm 1108$ & 0.002 \\
\hline
\end{tabular}


Table 4 (continued)

\begin{tabular}{lllll}
\hline Patient characteristic & RFCA cohort $(\mathrm{n}=40)$ & AF at follow-up $\mathrm{n}=10$ & SR at follow-up $\mathrm{n}=30$ & $\mathrm{p}$ value \\
\hline Number of energy application & $33 \pm 24$ & $56 \pm 32$ & $26 \pm 17$ & 0.005 \\
\hline
\end{tabular}

Values are mean \pm standard deviation or $\mathrm{n}(\%)$. Left ventricular ejection fraction was calculated by Simpson's method

$A C E$ angiotensin-converting enzyme, $A R B$ angiotensin receptor blocker, $B M I$ body mass index, $C R P$ C-reactive protein, $e G F R$ estimated glomerular filtration rate, INR international normalized ratio, $L A$ left atrium, TIA transient ischaemic attack

Normal values: haemoglobin $130-180 \mathrm{~g} / \mathrm{L}$ in men and $115-165 \mathrm{~g} / \mathrm{L}$ in women; haematocrit $40-52 \%$ in men and $36-47 \%$ in women; platelet count $150-400 \times 10^{9} / \mathrm{L}$; INR range $2.0-3.0$; creatinine $60-110 \mu \mathrm{mol} / \mathrm{L}$ in men and $45-90 \mu \mathrm{mol} / \mathrm{L}$ in women; eGFR: above $90 \mathrm{~mL} / \mathrm{min} / 1.73 \mathrm{~m}$; total cholesterol $<5.2 \mathrm{mmol} / \mathrm{L} ; \mathrm{CRP} 0-10 \mathrm{mg} / \mathrm{L}$; fibrinogen $2-5 \mathrm{~g} / \mathrm{L}$
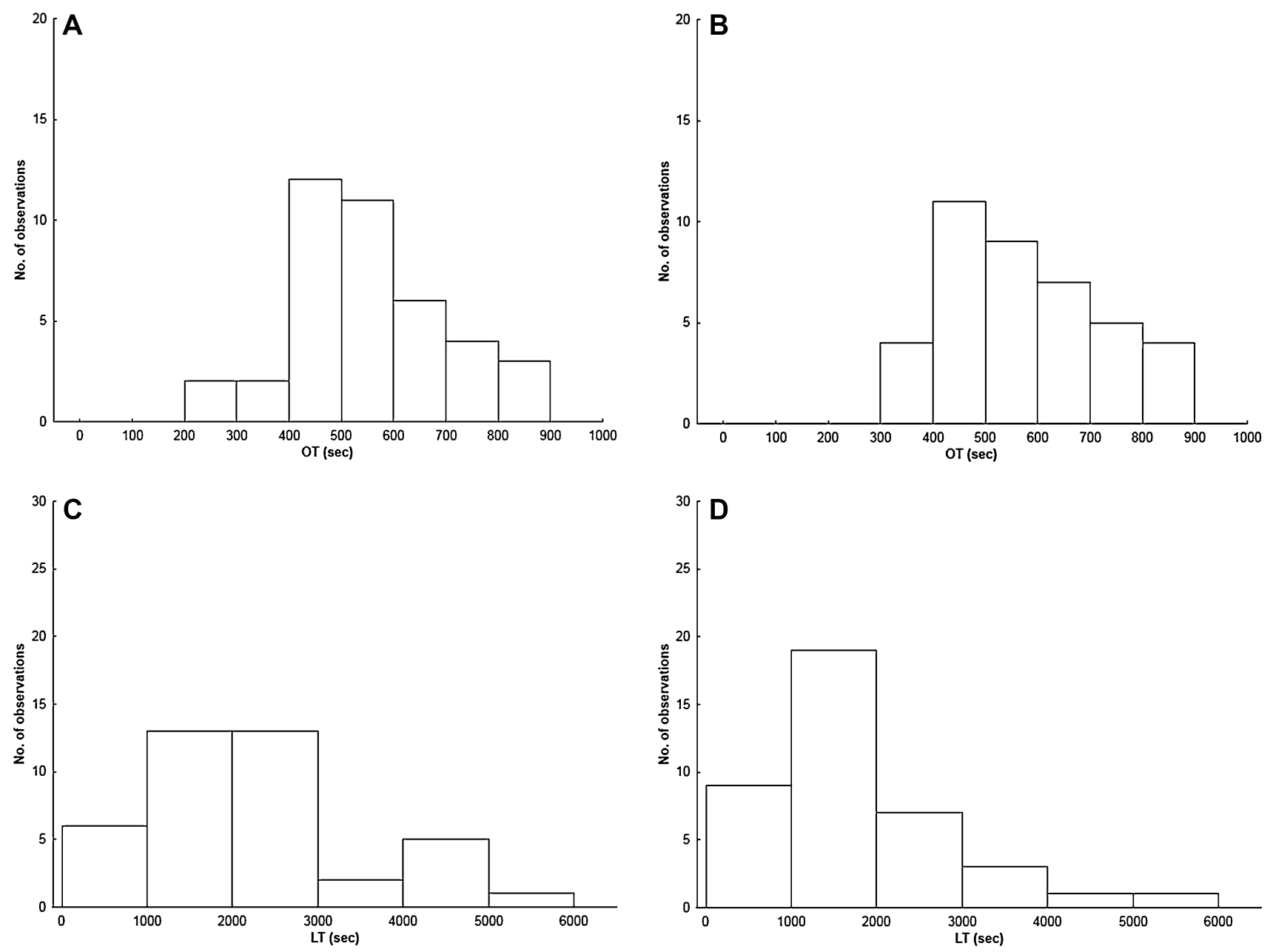

Fig. 1 Distribution of occlusion time (OT) before (a) and after radiofrequency catheter ablation (RFCA) (b); and distribution of lysis time (LT) before (c) and after RFCA (d). Y-axis shows number of observations (subjects)

standard practice, will help define the role of ablation in reducing adverse clinical outcomes.

The DCCV cohort was sampled at 6 weeks and the RFCA cohort at 3 months. 3 months is the conventional period to assess RFCA outcome; however, we arranged DCCV follow-up at 6 weeks because of concern that beyond this, few patients would still be in SR. Thus, sampling times are not directly comparable in the two groups and the lack of 

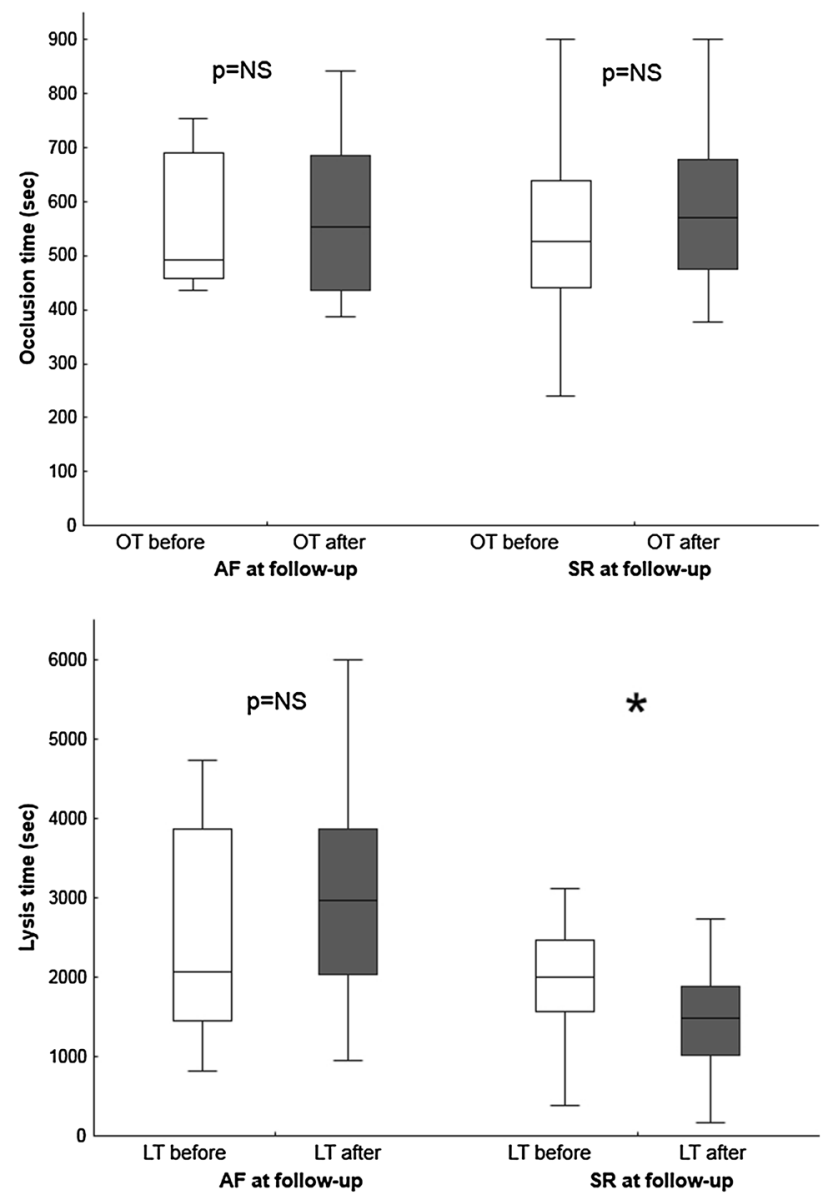

Fig. 2 Occlusion time (OT) and Lysis time (LT) before and after AF ablation, with respect to rhythm at follow-up. Sinus rhythm (SR) $* \mathrm{p}<0.001$

improvement in LT following DCCV could be attributed to "stunning", since it may take many weeks of restoration of mechanical contractility, not just electrical restoration of SR, to observe changes in thrombotic status. One could also postulate that the RFCA group may have more complete freedom from paroxysmal atrial arrhythmias. The role of inflammation in the genesis and perpetuation of $\mathrm{AF}$ is increasingly recognized, with increasing AF burden associated with increasing CRP $[3,14]$. High-sensitivity CRP decreases after successful RFCA for long-standing persistent $\mathrm{AF}$, suggesting that $\mathrm{AF}$ itself may cause an inflammatory response $[14,15]$. This may explain why RFCA but not DCCV improved thrombotic profile, although conflicts with the lack of change in CRP following successful RFCA in our cohort.

Our study showing impaired endogenous fibrinolysis in AF that is improved by restoration of SR, is supported by data on circulating D-dimer levels. The hypercoagulable state of AF is mirrored by increased levels of circulating D-dimer, a product of cross-linked fibrin degradation, reflecting activation of coagulation, fibrinolysis, or both. D-dimer levels are raised in AF and may identify those at high thromboembolic risk [3]. In small studies, both cardioversion and RFCA caused an early rise in D-dimer within 1-3 days, but the late effects of RFCA are less well documented. Lim showed that neither fibrinogen nor D-dimer levels fell 30 days after successful AF ablation, despite maintenance of SR, although a rise in fibrinogen level was associated with AF recurrence [16]. After successful AF ablation, some studies show CRP levels decrease significantly [15] whilst others show no change.

The lack of change in platelet reactivity (OT) in response to RFCA, conflicts with an earlier study where maintenance of SR 6 months after RFCA, was associated with reduced platelet activation and improvement in endothelial function [17]. However, whilst some studies have shown enhanced platelet activation in AF, others have not, or have documented only a local, and not systemic, phenomenon [2]. The role of platelet activation in AF therefore remains contentious.

Thrombotic status was similar in the left atrium and systemic circulation, and is at odds with some earlier work, showing that platelet reactivity was increased in the left atrium compared to the peripheral circulation $[18,19]$. However, impaired endogenous thrombolysis in the systemic circulation is supported by a large volume of published data on the importance of circulating D-dimer levels in AF.

Limitations of our study include the fact that the DCCV cohort was assessed at 6 weeks and the RFCA cohort at 3 months. Thus, any differences in thrombotic status between those who maintained SR after DCCV and after RFCA, may simply be due to timing, perhaps due to incomplete restoration of left atrial mechanical contractility. We chose to sample patients post-RFA at 3 months because this is conventionally regarded as the end of the "blanking period". During the first 3 months after AF ablation, the so-called "blanking period", a substantial proportion of patients experience early recurrences of atrial tachyarrhythmias [20,21]. Such early recurrences are thought to be due to transient local inflammatory states unassociated with the risk of later AF recurrence, and thus do not necessarily represent treatment failure [20]. It has, therefore, become common practice to consider a periprocedural blanking period, during which recurrences are considered nonspecific and do not prompt reintervention. The Heart Rhythm Society/European Heart Rhythm Association/European Cardiac Arrhythmia Society expert consensus recommends using 3 months as the conventional post-procedural blanking period, as recurrence within the first 3 months after ablation is not significantly associated with procedural success or failure as up to $65 \%$ of patients with AF recurrence in the first few months post-ablation will not have any further arrhythmias during long-term follow up [21]. However, the problem is that post-cardioversion, many 
Fig. 3 Upper panel change in lysis time before (a) and after (b) RFCA in individual patients, according to rhythm at follow-up. Lower panel change in lysis time before (a) and after (b) cardioversion in individual patients, according to rhythm at follow-up. Sinus rhythm (SR). $* \mathrm{p}<0.001$
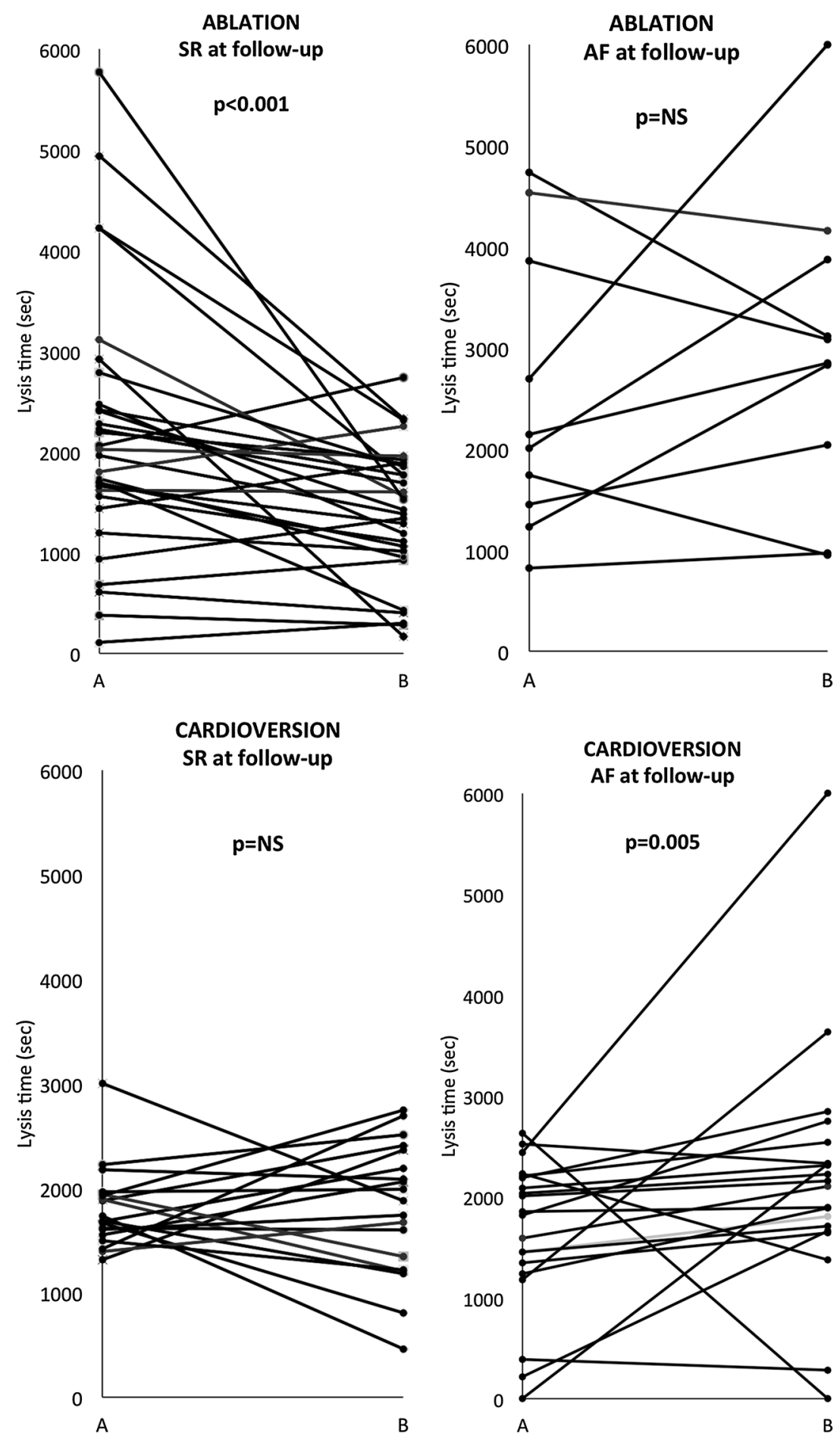

patients revert to AF by 3 months, so we had to bring forward the sampling point to 6 weeks post-DCCV to capture as many patients post-DCCV in sinus rhythm, as possible. Our DCCV and cardioversion cohorts were generally well matched but there were some differences, such as NOAC use. Another possible confounder is the difference in anticoagulant treatment type between the DCCV and the RFA groups. However, our study was done at a time when AF ablation was not routinely performed on NOACs and thus the sample population reflected clinical practice. However, whilst such differences in OAC may affect between group comparisons (between DCCV and RFA), it should not affect within-group comparisons (namely thrombotic status before and after DCCV, or before and after RFA) since patients acted as self-controls and continued on the same OAC treatment throughout. Furthermore, we recently compared the 

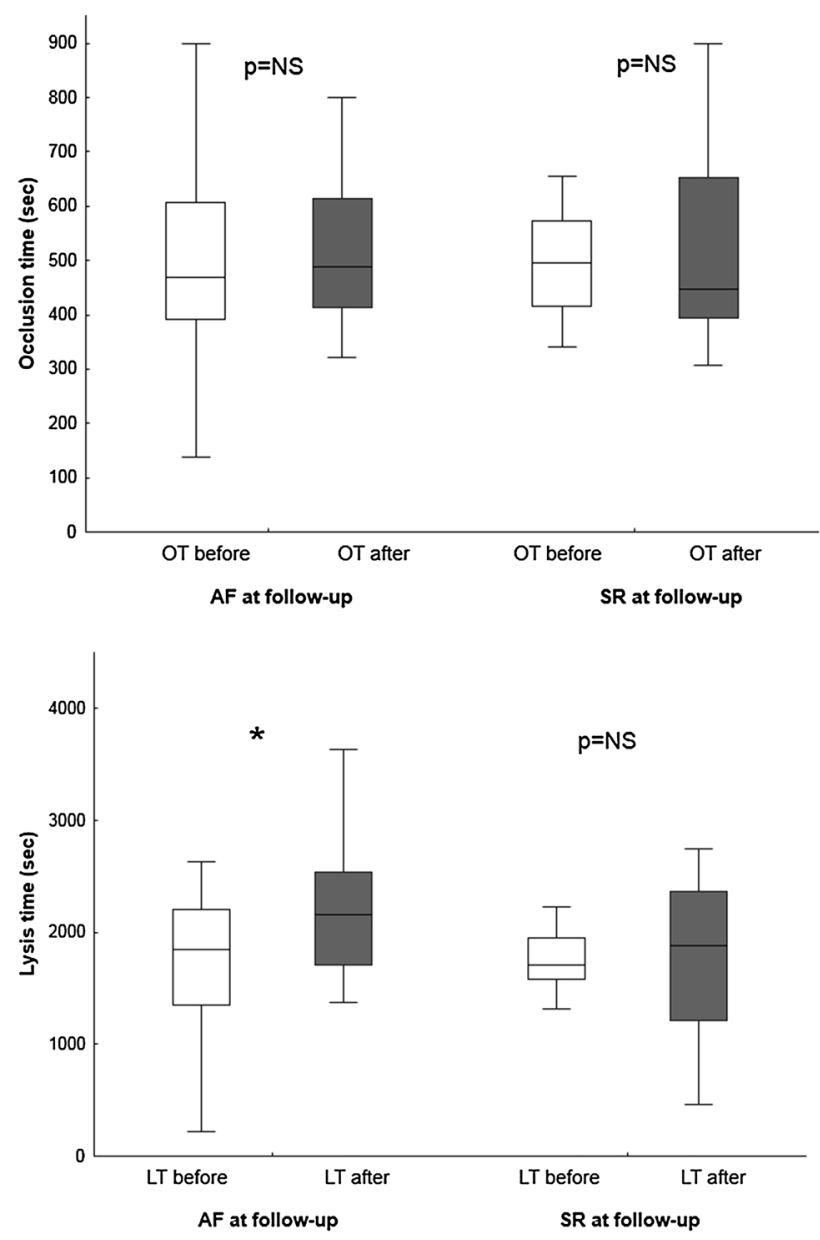

Fig. 4 Occlusion time (OT) before and after cardioversion, grouped with respect to rhythm at follow-up. Lysis time (LT) before and after cardioversion, grouped with respect to rhythm at follow-up. $* \mathrm{p}=0.005$. Sinus rhythm (SR)

relative effects of different non-vitamin $\mathrm{K}$ antagonist oral anticoagulants (rivaroxaban, dabigatran and apixaban) and warfarin, on global thrombotic status in patients with AF. We showed that NOACs and warfarin had a similar favourable effect on reducing platelet reactivity (evidenced by similar prolongation of OT with all agents). All NOACs exhibited a trend toward enhancing endogenous thrombolytic status (as evidenced by reduced LT), this was significant only for apixaban [22]. Therefore, although there are differences in the type of OAC used between the two cohorts, the impact of this on the results of thrombosis tests is likely to be non-significant.

Arrhythmia relapse reporting was based on symptoms and 24-h Holter monitor and/or follow-up ECG. It is thus possible that some patients experienced paroxysms of $\mathrm{AF}$ during follow-up but were misclassified as arrhythmia-free. Our patients were a mixed cohort of paroxysmal and persistent AF. Patients with paroxysmal AF may have intermediate levels of fibrin D-dimer compared to those with chronic AF or in SR which likely relates to fibrinolytic potential and thus the heterogeneous nature of our cohort may thus have been a potential confounder. However, against this is the finding that whilst the ratio of patients with paroxysmal AF was different between the DCCV and the RFA cohorts, OT and LT at baseline did not differ between the DCCV and RFA groups (Table 2). Furthermore, there was no difference in baseline OT or baseline LT between patients with different types of AF (paroxysmal, persistent, long-standing persistent). The GTT assesses platelet response to high shear, which does not replicate the low-flow state in the left atrium of patients with AF. However, unlike most low-flow states such as deep venous thrombosis where erythrocyte-rich thrombi prevail, in AF platelets contribute significantly to thrombus formation and the endothelial dysfunction which exists, suggests that an in vitro model like the GTT, where platelets and von Willebrand factor are key players and where endogenous fibrinolysis is assessed, may provide a useful assessment of this milieu. Furthermore, even if formed at low-flow states in the left atrium, such thrombi are subject to arterial flow, and our data and that of others suggest a systemic circulatory prothrombotic state and not just a localized left atrial phenomenon. The importance of endogenous fibrinolysis as a natural defence mechanism determining the outcome of thrombus formation is well recognized [23]. There are many physiological limitations to using single biomarkers to assess endogenous fibrinolysis, and emerging novel global assays of fibrinolysis such as used here have the potential to give a better overall assessment of thrombotic risk [23].

\section{Conclusion}

Successful restoration and maintenance of SR following RFCA of AF is associated with improved global thrombotic status as evidenced by enhanced endogenous fibrinolysis. Our pilot data indicate the need for future large studies to investigate whether maintenance of SR with RFCA can reduce thrombotic risk in patients with AF.

Funding Funded by R\&D Department of East and North Hertfordshire NHS Trust.

Author contributions MNS-data collection/acquisition, data analysis, drafting article, approval of final version. VM-research concept and design, data acquisition, data interpretation, critical revision, approval of final version, MF-data acquisition, data analysis, data interpretation, drafting article, approval of final version, DJdata acquisition, drafting article, approval of final version, TW-data acquisition, drafting article, approval of final version, DAG-research concept and design, data interpretation, critical revision, approval of final version. 
Open Access This article is distributed under the terms of the Creative Commons Attribution 4.0 International License (http://creativecommons.org/licenses/by/4.0/), which permits unrestricted use, distribution, and reproduction in any medium, provided you give appropriate credit to the original author(s) and the source, provide a link to the Creative Commons license, and indicate if changes were made.

\section{References}

1. Camm AJ, Lip GY, De Caterina R, Savelieva I, Atar D, Hohnloser SH, Hindricks G, Kirchhof P, Guidelines ESCCfP (2012) 2012 focused update of the ESC guidelines for the management of atrial fibrillation: an update of the 2010 ESC Guidelines for the management of atrial fibrillation. Developed with the special contribution of the European Heart Rhythm Association. Eur Heart J 33:2719-2747. doi:10.1093/eurheartj/ehs253

2. Khoo CW, Krishnamoorthy S, Lim HS, Lip GY (2012) Atrial fibrillation, arrhythmia burden and thrombogenesis. Int J Cardiol 157:318-323. doi:10.1016/j.ijcard.2011.06.088

3. Castellano JM, Chinitz J, Wilner J, Fuster V (2013) Mechanisms of stroke in atrial fibrillation. Cardiac Electrophysiol Clin 6:5-15

4. Vaidya K, Arnott C, Russell A, Masson P, Sy RW, Patel S (2015) Pulmonary vein isolation compared to rate control in patients with atrial fibrillation: a systematic review and meta-analysis. Heart Lung Circ 24:744-752. doi:10.1016/j.hlc.2015.02.025

5. Bunch TJ, May HT, Bair TL, Weiss JP, Crandall BG, Osborn JS, Mallender C, Anderson JL, Muhlestein BJ, Lappe DL, Day JD (2013) Atrial fibrillation ablation patients have long-term stroke rates similar to patients without atrial fibrillation regardless of CHADS2 score. Heart Rhythm 10:1272-1277. doi:10.1016/j. hrthm.2013.07.002

6. Wyse DG, Waldo AL, DiMarco JP, Domanski MJ, Rosenberg Y, Schron EB, Kellen JC, Greene HL, Mickel MC, Dalquist JE, Corley SD, Atrial Fibrillation Follow-up Investigation of Rhythm Management I (2002) A comparison of rate control and rhythm control in patients with atrial fibrillation. N Engl J Med 347:18251833. doi:10.1056/NEJMoa021328

7. Corley SD, Epstein AE, DiMarco JP, Domanski MJ, Geller N, Greene HL, Josephson RA, Kellen JC, Klein RC, Krahn AD, Mickel M, Mitchell LB, Nelson JD, Rosenberg Y, Schron E, Shemanski L, Waldo AL, Wyse DG, Investigators A (2004) Relationships between sinus rhythm, treatment, and survival in the Atrial Fibrillation Follow-Up Investigation of Rhythm Management (AFFIRM) Study. Circulation 109:1509-1513. doi:10.1161/01. CIR.0000121736.16643.11

8. Bunch TJ, Crandall BG, Weiss JP, May HT, Bair TL, Osborn JS, Anderson JL, Muhlestein JB, Horne BD, Lappe DL, Day JD (2011) Patients treated with catheter ablation for atrial fibrillation have long-term rates of death, stroke, and dementia similar to patients without atrial fibrillation. J Cardiovasc Electrophysiol 22:839-845. doi:10.1111/j.1540-8167.2011.02035.x

9. Marin F, Roldan V, Lip GY (2003) Fibrinolytic function and atrial fibrillation. Thromb Res 109:233-240

10. Kamath S, Blann AD, Chin BS, Lip GY (2002) A prospective randomized trial of aspirin-clopidogrel combination therapy and dose-adjusted warfarin on indices of thrombogenesis and platelet activation in atrial fibrillation. J Am Coll Cardiol 40:484-490

11. Saraf S, Christopoulos C, Salha IB, Stott DJ, Gorog DA (2010) Impaired endogenous thrombolysis in acute coronary syndrome patients predicts cardiovascular death and nonfatal myocardial infarction. J Am Coll Cardiol 55:2107-2115. doi:10.1016/j. jacc.2010.01.033

12. Noseworthy PA, Kapa S, Deshmukh AJ, Madhavan M, Van Houten H, Haas LR, Mulpuru SK, McLeod CJ, Asirvatham
SJ, Friedman PA, Shah ND, Packer DL (2015) Risk of stroke after catheter ablation versus cardioversion for atrial fibrillation: a propensity-matched study of 24,244 patients. Heart Rhythm 12:1154-1161. doi:10.1016/j.hrthm.2015.02.020

13. Kornej J, Lip GY, Bollmann A (2013) Reduction of stroke and mortality in patients with atrial fibrillation by catheter ablation? Finally, tackling the hard endpoints. Europace: European pacing, arrhythmias, and cardiac electrophysiology: journal of the working groups on cardiac pacing, arrhythmias, and cardiac cellular electrophysiology of the European. Soc Cardiol 15:620-622. doi:10.1093/europace/eus389

14. Guo Y, Lip GY, Apostolakis S (2012) Inflammation in atrial fibrillation. J Am Coll Cardiol 60:2263-2270. doi:10.1016/j. jacc.2012.04.063

15. Rotter M, Jais $P$, Vergnes MC, Nurden $P$, Takahashi $Y$, Sanders $P$, Rostock T, Hocini M, Sacher F, Haissaguerre M (2006) Decline in $\mathrm{C}$-reactive protein after successful ablation of long-lasting persistent atrial fibrillation. J Am Coll Cardiol 47:1231-1233. doi:10.1016/j.jacc.2005.12.038

16. Lim HS, Schultz C, Dang J, Alasady M, Lau DH, Brooks AG, Wong CX, Roberts-Thomson KC, Young GD, Worthley MI, Sanders P, Willoughby SR (2014) Time course of inflammation, myocardial injury, and prothrombotic response after radiofrequency catheter ablation for atrial fibrillation. Circ Arrhythm Electrophysiol 7:83-89. doi:10.1161/CIRCEP.113.000876

17. Lim HS, Willoughby SR, Schultz C, Chakrabarty A, Alasady M, Lau DH, Roberts-Thomson KC, Worthley MI, Young GD, Sanders P (2014) Successful catheter ablation decreases platelet activation and improves endothelial function in patients with atrial fibrillation. Heart Rhythm 11:1912-1918. doi:10.1016/j. hrthm.2014.07.030

18. Willoughby SR, Roberts-Thomson RL, Lim HS, Schultz C, Prabhu A, De Sciscio P, Wong CX, Worthley MI, Sanders P (2010) Atrial platelet reactivity in patients with atrial fibrillation. Heart Rhythm 7:1178-1183. doi:10.1016/j.hrthm.2010.01.042

19. Akar JG, Jeske W, Wilber DJ (2008) Acute onset human atrial fibrillation is associated with local cardiac platelet activation and endothelial dysfunction. J Am Coll Cardiol 51:1790-1793. doi:10.1016/j.jacc.2007.11.083

20. Willems S, Khairy P, Andrade JG, Hoffmann BA, Levesque S, Verma A, Weerasooriya R, Novak P, Arentz T, Deisenhofer I, Rostock T, Steven D, Rivard L, Guerra PG, Dyrda K, Mondesert B, Dubuc M, Thibault B, Talajic M, Roy D, Nattel S, Macle L, Investigators* AT (2016) Redefining the blanking period after catheter ablation for paroxysmal atrial fibrillation: insights from the ADVICE (adenosine following pulmonary vein isolation to target dormant conduction elimination) trial. Circ Arrhythm Electrophysiol 9. doi:10.1161/CIRCEP.115.003909

21. Calkins H, Kuck KH, Cappato R, Brugada J, Camm AJ, Chen SA, Crijns HJ, Damiano RJ Jr, Davies DW, DiMarco J, Edgerton J, Ellenbogen K, Ezekowitz MD, Haines DE, Haissaguerre M, Hindricks G, Iesaka Y, Jackman W, Jalife J, Jais P, Kalman J, Keane D, Kim YH, Kirchhof P, Klein G, Kottkamp H, Kumagai K, Lindsay BD, Mansour M, Marchlinski FE, McCarthy PM, Mont JL, Morady F, Nademanee K, Nakagawa H, Natale A, Nattel S, Packer DL, Pappone C, Prystowsky E, Raviele A, Reddy V, Ruskin JN, Shemin RJ, Tsao HM, Wilber D (2012) 2012 HRS/ EHRA/ECAS expert consensus statement on catheter and surgical ablation of Atrial Fibrillation: recommendations for patient selection, procedural techniques, patient management and follow-up, definitions, endpoints, and research trial design. Europace: European pacing, arrhythmias, and cardiac electrophysiology: journal of the working groups on cardiac pacing, arrhythmias, and cardiac cellular electrophysiology of the European Society of Cardiology. Europace 14:528-606. doi:10.1093/europace/eus027 
22. Farag M, Niespialowska-Steuden M, Okafor O, Artman B, Srinivasan M, Khan A, Sullivan K, Wellsted D, Gorog DA (2016) Relative effects of different non-vitamin K antagonist oral anticoagulants on global thrombotic status in atrial fibrillation. Platelets 27:687-693. doi:10.3109/09537104.2016.1158402
23. Okafor ON, Gorog DA (2015) Endogenous fibrinolysis: an important mediator of thrombus formation and cardiovascular risk. $\mathrm{J}$ Am Coll Cardiol 65:1683-1699. doi:10.1016/j.jacc.2015.02.040 